\title{
Hypoxia and human disease - and the Journal of Molecular Medicine
}

\author{
G. L. Semenza
}

Received: 29 October 2007 / Accepted: 29 October 2007 / Published online: 20 November 2007

(C) Springer-Verlag 2007

This special issue of the Journal of Molecular Medicine is focused on the role of hypoxia in the pathogenesis of human disease. Oxygen homeostasis is a critical organizing principle of metazoan evolution, development, and physiology. The seven invited reviews in this issue highlight the rapid progress that is being made in delineating the molecular mechanisms that result in disruption of oxygen homeostasis in different disease states, the extent to which this disruption contributes to disease pathogenesis, and the prospects for novel therapies that target pathways involved in adaptive (or maladaptive) responses to hypoxia. In their review, entitled "Hypoxia and Gastrointestinal Disease," Cormac Taylor and Sean Colgan summarize the results of published studies analyzing hypoxia-induced gene expression in intestinal epithelial cells and the role of hypoxia in the pathogenesis of inflammatory bowel disease. In "Hypoxia and Cancer," Christiane Brahimi-Horn, Johanna Chiche, and Jacques Pouyssegur discuss the causes and consequences of intratumoral hypoxia. Joseph Garcia discusses ischemic heart disease with a specific focus on alterations in cardiac metabolism that promote the survival of ischemic cells. In "Hypoxia and Chronic Lung Disease," Rubin Tuder and colleagues review the role of hypoxia in respiratory distress syndrome, high altitude pulmonary edema, pulmonary arterial hypertension, and chronic obstructive pulmonary disease. It is remarkable that despite receiving oxygen from the bronchial circulation and directly from the alveolar air sacs, hypoxia can develop within the lungs (even at sea level) and contribute to

\section{G. L. Semenza $(\square)$}

Vascular Program, Institute for Cell Engineering,

The Johns Hopkins University School of Medicine,

Broadway Research Building, Suite 671, 733 North Broadway,

Baltimore, MD 21205, USA

e-mail: gsemenza@jhmi.edu pathogenic tissue remodeling. Masaomi Nangaku and Kai-Uwe Eckardt discuss the role of the kidney in oxygen homeostasis, particularly in the production of erythropoietin, which is the glycoprotein hormone that controls red blood cell production. They also summarize recent data indicating that chronic hypoxia in the tubulointerstitium represents a final common pathway to end-stage kidney disease. Rajiv Ratan and his colleagues discuss novel strategies for promoting hypoxic adaptation in neurons as a means to prevent or treat brain injury after stroke. These strategies are based on recent advances in our understanding of the molecular physiology of oxygen sensing. Annelies Zinkernagel, Randall Johnson, and Victor Nizet review data indicating that the same transcriptional regulatory system that promotes adaptive responses to hypoxia, which are described in the other six papers in this issue, is also required for neutrophil inflammatory and innate immune functions. They also review data indicating that obligate intracellular bacteria and oncogenic viruses modulate hypoxia response pathways in host cells.

Hypoxia-inducible factor 1 (HIF-1) is a transcription factor that plays an important role in cellular and systemic responses to reduced oxygen availability in all metazoan species. Purification of HIF-1 from human cells revealed that it is heterodimer composed of HIF-1 $\alpha$ and HIF-1 $\beta$ subunits, which dimerize and bind to DNA containing the core sequence $5^{\prime}-(\mathrm{A} / \mathrm{G}) \mathrm{CGTG}-3^{\prime}$. Database searches for proteins that were homologous to HIF- $1 \alpha$ led to the identification of HIF- $2 \alpha$, which can also dimerize with HIF- $1 \beta$ and bind to an overlapping but distinct set of target genes. HIF- $1 \alpha$ and HIF- $2 \alpha$ are each negatively regulated by $\mathrm{O}_{2}$-dependent hydroxylation of key proline and asparagine residues that dramatically reduce protein half-life and transcriptional activity, respectively, under aerobic conditions. Although I admit my bias in focusing on the role of 
HIF-1 in disease pathogenesis, the seven outstanding papers in this issue provide abundant evidence that this line of investigation is leading to greater insight into the pathogenesis of gastrointestinal, neoplastic, cardiac, pulmonary, renal, cerebral, and infectious diseases. As discussed in several of the reviews in this issue, novel strategies that increase HIF-1 activity, either by gene therapy or by pharmacologic inhibition of hydroxylase activity, may provide therapeutic benefit in patients with heart, inflammatory bowel, and kidney diseases, whereas inhibitors of HIF-1 may be useful as anti-cancer agents.

This special issue of the Journal of Molecular Medicine was designed with two goals in mind. First, we sought to highlight one of the most dynamic areas of contemporary biomedical research and to illustrate the extent to which disruption of oxygen homeostasis represents a key element in the pathogenesis of the common causes of mortality in the industrialized world. Indeed, this issue has seven papers by virtue of editorial, not scientific, limitations, and we could easily have covered twice as many areas of biomedical research. Similarly, the role of pathways other than those involving HIF-1 and its hydroxylases could fill another issue. The role in hypoxia in other human diseases and other signaling pathways will be covered in future issues of the journal. This issue could not have been produced without the generous assistance of expert reviewers who were willing to provide helpful comments on very short deadlines, and we would like to thank Valina Dawson, Serpil Erzurum, Amato Giaccia, Mark Gillespie, Volker Haase, Jan-Steffen Juergensen, Victor Nizet, Nanduri Prabhakar, and Mikhail Sitkovsky for their efforts.

The second major purpose for producing this special issue was to encourage investigators studying the role of hypoxia in human disease to view the Journal of Molecular Medicine as a preferred vehicle for dissemination of scientific advances in this exciting area of biomedical research. I am grateful to Detlev Ganten for the opportunity to work with him as Editor in Chief of the Journal of Molecular Medicine and for his enthusiastic support of this new focus on oxygen-related pathobiology. To support this new focus, our illustrious team of Associate Editors has been expanded by the addition of Stylianos Antonarakis (Genetics), Valina Dawson (Neuroscience), Jun Liu (Pharmacology and Signal Transduction), Giovanni Melillo (Oncology), Victor Nizet (Immunology and Infectious Disease), and Harald Schmidt (Cardiovascular Pharmacology). With the help of all of our Associate Editors around the world, along with Managing Editor Christiane Nolte and the Editorial Office staff in Berlin, the Journal of Molecular Medicine will strive to provide rapid, objective, and constructive critiques of all submitted manuscripts through the online Manuscript Central submission system, which can be accessed at http://mc.manuscriptcentral.com/jmm. For now, take a deep breath and dive into this exciting issue. 